\title{
Dynamics of $\mathbf{N}_{2}$ sticking on $\mathrm{W}(100)$ : The decisive role of van der Waals interactions ${ }^{\dagger}$
}

\author{
Alejandro Peña-Torres, ${ }^{* a b}$ H. Fabio Busnengo, ${ }^{c}$ J. Iñaki Juaristi, ${ }^{\text {bde }}$ Pascal Larregaray, ${ }^{a f}$ \\ and Cédric Crespos ${ }^{a f}$
}

Received Date

Accepted Date

DOI: $10.1039 / x x x x x x x x x x$

www.rsc.org/journalname

The reactive dynamics of $\mathrm{N}_{2}$ on $\mathrm{W}(100)$ has been investigated by means of quasi-classical trajectory calculations using an interpolated six-dimensional potential energy surface (PES) based on density functional theory energies obtained employing the vdW-DF2 functional. The dynamics are compared to those obtained using the PW91 functional and to experimental data. The results show that the new PES provides a significant improvement in the description of the reactivity in this system. We show that the long standing problem that constituted the large qualitative disagreement between the simulations performed with the PW91-PES and the experiments was due to the presence of energy barriers in the entrance channel that disappear when vdW forces are accounted for.

Over the last decades, the interaction of diatomic molecules with metallic surfaces has been thoroughly studied, seeking a fundamental understanding of the simplest type of molecule-metal reactions in light of their important role in heterogeneous chemistry $1-5$. Molecular beams experiments 6 - 11 and theoretical studies ${ }^{12}$ 17 have been working together to analyze in details the gassurface reactions at molecular level.

Most of the theoretical simulations are done within the Born-

\footnotetext{
a Université Bordeaux, ISM, UMR5255, F-33400 Talence, France. E-mail: apenat.un@gmail.com

${ }^{b}$ Centro de Física de Materiales CFM/MPC (CSIC-UPV/EHU), Paseo Manuel de Lardizabal 5, 20018 Donostia-San Sebastián, Spain.

${ }^{c}$ Instituto de Física de Rosario (CONICET-UNR) and Facultad de Ciencias Exactas, Ingeniería y Agrimensura, Universidad Nacional de Rosario, Avenida Pellegrini 250, 2000 Rosario, Argentina.

${ }^{d}$ Departamento de Física de Materiales, Facultad de Químicas (UPV/EHU), Apartado 1072, 20080 Donostia-San Sebastián, Spain.

e Donostia International Physics Center (DIPC), Paseo Manuel de Lardizabal 4, 20018 Donostia-San Sebastián, Spain.

${ }^{f}$ CNRS, ISM, UMR5255, F-33400 Talence, France.

$\dagger$ Electronic Supplementary Information (ESI) available: Full curve of the probability for the $\mathrm{N}_{2}$ molecules to reach a distance of $\mathrm{Z}=2.5 \AA$ from the surface as a function of the collision energy. See DOI: 10.1039/cXCP00000x/
}

Oppenheimer approximation, making use of potential energy surfaces (PES) based on density functional theory (DFT) and the generalized gradient approximation (GGA) for the exchangecorrelation (EXC) functional. Within this level of theory different phenomena including reactivity $18-23$ and also scattering properties such as diffraction peak positions ${ }^{18 \mid 24}$ and angular $21 \mid 25$, internal state 26,29 and energy distributions $25 \mid 30$ of the scattered particles, have been reasonably well described for a variety of molecule-surface combinations.

In this respect, one of the long-lasting problems has been the description of the dissociative adsorption of $\mathrm{N}_{2}$ on the $\mathrm{W}(100)$ surface, which is known to be significantly more reactive at low initial kinetic energies than the $\mathrm{W}(110)$ face 31 . The prominent crystallographic anisotropy effect on the $\mathrm{N}_{2}$ dissociation probability has been studied in the past ${ }^{32}$ by means of dynamics simulations performed under normal incidence on a DFT PES obtained using the PW91 exchange-correlation potential 33 . The results were subsequently compared to molecular beams experimental data 34 37. The simulations were successful in reproducing the nonactivated character of dissociative adsorption in the W(100) surface, which manifests in the high adsorption probability $S_{0}$ at incidence energies below $E_{i} \simeq 10 \mathrm{meV}$. However, theory greatly overestimates the measured $\mathrm{S}_{0}$ for $E_{i}>200 \mathrm{meV}$ and shows a non-monotonic dependency of $\mathrm{S}_{0}$ below $500 \mathrm{meV}$ incident energy, including a steep decrease up to $E_{i} \simeq 40 \mathrm{meV}$, an increase in the $40 \mathrm{meV}<E_{i}<200 \mathrm{meV}$ range with a peak at the latest energy, and a monotonic decrease for $E_{i}>200 \mathrm{meV}$. No such intricate behavior was observed in the experiments in which $S_{0}$ decreases steeply up to value of $E_{i} \simeq 500 \mathrm{meV}$ that depends slightly on the surface temperature, and increases slowly with $E_{i}$ further on $35 \mid 36$. It is worth noting that dynamics simulations accounting for electronic excitations within the local density friction approximation (LDFA) 38 showed that $S_{0}$ is not much affected by nonadiabatic effects in this system 39 , therefore, could not resolve the disagreement between theory and experiments.

The central approximation in the construction of a DFT-PES re- 
lies on the choice of the EXC functional. For this reason, when discrepancies are found between the simulations and the experiments, the performance of the chosen EXC functional for the specific system should be put into question. For instance, though for the $\mathrm{N}_{2} / \mathrm{W}(110)$ the deviations between theory and experiment were not as severe as in the present case ${ }^{31 / 40 / 41}$, this argument led to the use of different GGA-EXC functionals for this system ${ }^{2642}$. These studies showed that, certainly, the dynamics were sensitive to the choice of the functional, but no clear conclusions were made about the better functional since, for instance, the RPBE ${ }^{43}$ seemed to work better for normal incidence conditions whereas the PW91 ${ }^{33}$ exhibited better agreement with the experiments at off-normal incidence. More recently, and also for $\mathrm{N}_{2} / \mathrm{W}(110)$, using $A b$ Initio Molecular Dynamics (AIMD) simulations, the performance of non-local functionals that account for long range van der Waals (vdW) interactions ${ }^{44 / 45}$ has been tested. Though the dynamics were indeed affected by the use of vdW functionals, no clear improvement over the results obtained with the GGAs was observed when comparing with the experimental data. However, a recent study has shown that the dynamics performed in a DFT-PES based on a vdW EXC functional for the $\mathrm{CO} / \mathrm{Ru}(0001)$ system achieve excellent agreement with experiments for the adsorption probabilities and for the angular and energy distributions of the scattered particles 46 49.

Motivated by these results, we have built a new DFT-PES based on the vdW-DF $2^{50}$ EXC functional for the $\mathrm{N}_{2} / \mathrm{W}(100)$ system, for which we have performed molecular dynamics simulations. The results for $S_{0}$ are compared to those obtained by Volpilhac et al. ${ }^{32}$ with the PW91-PES using the Born-Oppenheimer static surface approximation (BOSS), in order to confirm that the above discussed intricate dependency with energy of $S_{0}$ not observed in the experiments is a result of the neglect of long range vdW forces by the semi-local GGA functional. For a better comparison with experiments, energy exchange between the molecules and the surface phonons has been taken into account through the use of the generalized Langevin oscillator model (GLO) $51+53$. The results show that by using the new vdW-DF2-PES a semi-quantitative agreement with experiments is obtained for the dependency of $S_{0}$ on both the incident energy of the molecule and the temperature of the surface.

A brief description of the theoretical methodology follows. To obtain the six-dimensional PES for the $\mathrm{N}_{2}+\mathrm{W}(100)$ system, we have performed a set of DFT calculations using the vdW-DF2 functional as implemented in the VASP code ${ }^{54}$. A periodic supercell was used where the metallic surface is represented by a 5-layer slab, and the calculations were done using a $8 \times 8 \times 1$ MonkhorstPack grid of $k$ points for a $(2 \times 2)$ structure with a lattice constant $a=3.24 \AA$. Considering that the experiments to which our results are here compared were performed at temperatures above 200 $\mathrm{K}$, the structural phase transition leading to a $\mathrm{c}(2 \times 2)$ zigzag surface atom rearrangement at lower temperatures ${ }^{55[56}$ has been ignored. A total number of 11870 DFT energies were calculated for the construction of the multidimensional PES, corresponding to 35 configurations of the $\mathrm{N}_{2}$ molecule over the surface. Each configuration is defined by the position of the center of mass of the molecule in cartesian coordinates $(X, Y, Z)$, the N-N distance $(\mathrm{r})$

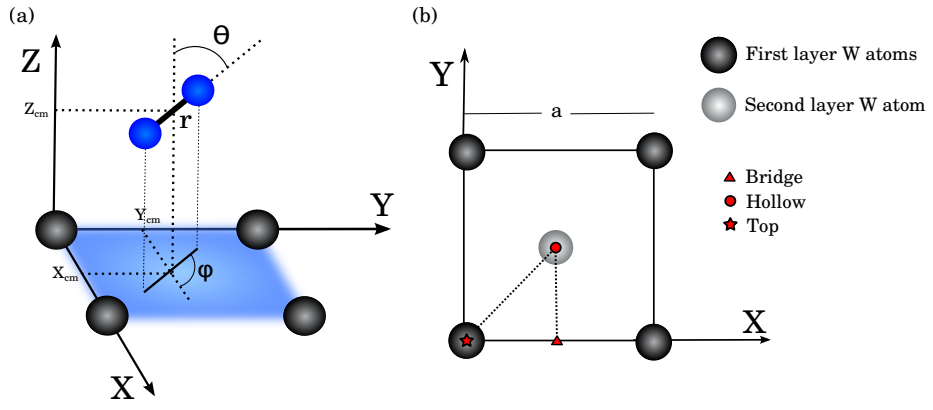

Fig. 1 (a) Coordinate system used for the calculations, nitrogen atoms are in blue and surface $W$ atoms are in gray. The Cartesian frame origin is located on a $W$ atom. $Z$ is the direction normal to the surface [100], and $X$ and $Y$ are the directions parallel to the surface [010] and [001], respectively. (b) Top-view of the $\mathrm{W}(100)$ unit cell, high symmetry points are marked.

and the orientation with respect to the surface in terms of the po$\operatorname{lar}(\theta)$ and azimuthal $(\phi)$ angles (See Fig. 11(a)). For each configuration, we have calculated an 11-point grid in $r$ space (0.7-2.3 $)$ and a 17-point grid in $Z$ space $(0.5-7.5 \AA)$ while keeping fixed the other degrees of freedom (i.e. X, Y, $\theta, \phi$ ). The evaluation of any point on the $6 \mathrm{D}$ PES is achieved through interpolation using the Corrugation Reducing Procedure (CRP) ${ }^{57+59}$ assuming that surface atoms are kept at their equilibrium positions. As stated before, dynamics of $\mathrm{N}_{2}$ on the $\mathrm{W}(100)$ surface were studied performing quasi-classical trajectory calculations on the single adiabatic 6D-PES.

First of all we proceed to perform a static analysis of the main features of the new vdW-DF2-PES as compared to the PW91-PES of Volpilhac et al.32. In particular, we focus on the two distinct nonactivated paths to dissociation identified in that reference. At distances from the surface of around $3 \AA<Z<4 \AA$ the PW91-PES presents energy barriers for most of the molecular configurations. At these distances, the only barrierless configurations in which the molecules can approach the surface are those corresponding to the center of mass (CM) located around the top site with an orientation of the molecular axis close to vertical $(X=0 ; Y=0$; $\theta=0^{\circ}$ ). Afterwards the nonactivated path leads to configurations with the $\mathrm{CM}$ over the bridge site and the molecular axis parallel to the surface $\left(X=a / 2 ; Y=0 ; \theta=90^{\circ}\right)$. At this point, the two mentioned dissociation paths separate. If the molecule axis is perpendicular to the W-W bond $\left(\phi=90^{\circ}\right)$ they found that the molecules can dissociate without barriers in this configuration. If the molecular axis is parallel to the W-W bond $\left(\phi=0^{\circ}\right)$ a second nonactivated path to dissociation existed in which the molecules first travel to the hollow site $(X=a / 2 ; Y=a / 2)$ where they subsequently dissociate. Interestingly, whereas in our vdW-DF2-PES this second path is still nonactivated, this is not longer the case in the former path with $\phi=90^{\circ}$ for which energy barriers to dissociation of the order of $\simeq 650 \mathrm{meV}$ appear. The reduction of the number of nonactivated pathways to dissociation that the $\mathrm{N}_{2}$ molecules can follow in the vdW-DF2-PES is expected to lower the reaction probabilities. The more repulsive character at short distances from the surface of the vdW-DF2-PES as compared to the PW91-PES can be also viewed in Table 1 In this table, potential 
Table 1 Comparison of the potential minima for both PESs at a given configuration of the $\mathrm{N}_{2}$ molecule over the high symmetry sites of the unit cell (See the Supporting Information, Figure S1). For each site, the distance to the surface $(Z)$ and the internuclear distance $(r)$ corresponding to the minimum are presented

\begin{tabular}{lcccc}
\hline & \multicolumn{4}{c}{ PM $(\mathrm{eV})^{h}$} \\
\cline { 2 - 5 } Top & \multicolumn{2}{c}{ PW91-PES } & \multicolumn{2}{c}{ vdW-DF2-PES } \\
\hline \multirow{2}{*}{ Bridge } & -1.09 & $\begin{array}{l}\mathrm{Z}=2.60 \AA \\
\mathrm{r}=1.14 \AA\end{array}$ & -0.746 & $\begin{array}{l}\mathrm{Z}=2.68 \AA \\
\mathrm{r}=1.13 \AA\end{array}$ \\
\hline Hollow & -2.15 & $\begin{array}{l}\mathrm{Z}=1.72 \AA \\
\mathrm{r}=1.23 \AA\end{array}$ & -0.654 & $\begin{array}{l}\mathrm{Z}=1.82 \AA \\
\mathrm{r}=1.97 \AA \\
\mathrm{r}=1.42 \AA\end{array}$ \\
\hline
\end{tabular}

energies of local minima along the minimun energy reaction path have been compared. ${ }^{g}$ At any given high symmetry site, potential minima are lower in the PW91-PES case. It is also noteworthy that the there is no significant displacement in the position of minima from one functional to the other, since only a shift of less than $0.1 \AA$ in $Z$ and $r$ is observed.

To understand better the impact of these changes in the PESs on the adsorption of $\mathrm{N}_{2}$, results of quasi-classical dynamics calculations under normal incidence are reported on Fig. 2. First, a comparison of the sticking coefficient $\mathrm{S}_{0}$ as a function of the $\mathrm{N}_{2}$ initial kinetic energy $E_{i}$ for both PESs is plotted (see left panel of Fig. 2), the results are obtained under the BOSS approximation employing a Monte Carlo sampling for the initial conditions of the 10000 trajectories for each value of impact energy. Sticking coefficient is defined as the probability for the molecules to remain at the surface without being reflected (if $P_{r e f}$ is defined as the reflection probability, $S_{0}=1-P_{\text {ref }}$ ). Thus, sticking involves both dissociated and molecularly adsorbed molecules on the surface, however, for the case of BOSS calculations it accounts almost entirely for dissociated molecules, given that virtually no molecular trapping ( $\leq 1 \%$ when $E_{i} \simeq 10 \mathrm{meV}$ ) is observed after the integration time (50 ps). As our static analysis suggested, we observe that the vdW-DF2-PES is much less reactive than the PW91-PES. Common to the two PESs, a high value of the sticking coefficient is observed at almost zero collision energy ( $E_{i} \simeq 10 \mathrm{meV}$ ), followed by a steep decrease of $S_{0}$ at very low collision energy $(10 \mathrm{meV}$ $<E_{i}<40 \mathrm{meV}$ ). Beyond $40 \mathrm{meV}$ the shape of $S_{0}$ is rather different from one PES to the other. As pointed out before, the PW91PES exhibits an increase in the sticking probability with a peak at about $200 \mathrm{meV}$, whereas the vdW-DF2-PES is characterized by a monotonic decay of $S_{0}$ with energy, result that agrees quite well with experiments.

In order to study further the non-monotonic behavior of $\mathrm{S}_{0}$ with

\footnotetext{
${ }^{g}$ We note that these local minima of the PES located along the minimum energy path to dissociation are minima in 2 dimensions $(r, Z)$ except the configuration at the top site which is a real minimum in the 6 dimensional configurational space. It is worth to mention that there exists another $6 D$ minimum that corresponds to the molecule on the hollow site parallel to the surface and with $\phi=45^{\circ}$ (See Supplemental Information) which is, actually, the minimum energy configuration for the non-dissociated molecule but that is not located in the minimum energy path to dissociation.

${ }^{h}$ Potential Minima: For the top site, $\theta=0^{\circ}$, for the bridge and hollow sites, $\theta=90^{\circ}$ and $\phi=0^{\circ}$
}
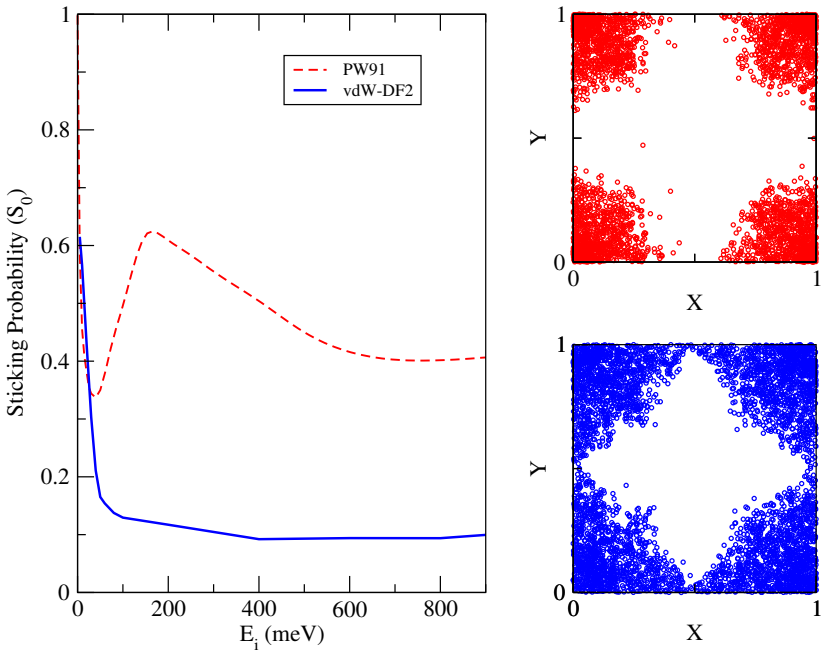

Fig. 2 Total sticking probability for $\mathrm{N}_{2}$ molecules on $\mathrm{W}(100)$ under normal incidence as a function of the collision energy $\left(E_{i}\right)$ obtained with functionals PW91 (dashed red) and vdW-DF2 (solid blue) within the BOSS approximation (left panel). Location of the molecular center of mass for 10000 trajectories over the unit cell when the molecule reaches a distance of $Z=2.5 \AA$ to the surface with an impact energy of $40 \mathrm{meV}$. $X$ and $Y$ coordinates are given in units of the lattice constant $a$ for the PW91PES (upper right panel) and vdW-DF2-PES (bottom right panel). The W atoms are located at the corners of the plots.

energy when the PW91-PES is used, we focus on the case close to $40 \mathrm{meV}$ collision energy for which the minimum of $S_{0}$ is found (See the Supporting Information, Figure S2). On the right side of Fig. 2 we have plotted the distribution in $X$ and $Y$ of the molecular center of mass for the trajectories reaching an altitude of $2.5 \AA$ over the surface out of a set of 10000 trajectories for both PESs. The upper panel corresponds to the results obtained with the PW91-PES, for which $67.6 \%$ of the trajectories are able to reach this distance. For the case of the vdW-DF2-PES (bottom panel) this number is much larger and amounts up to $92.9 \%$. Moreover, and looking at the plots, we find that the molecules reaching this distance for the PW91-PES are located in a far more confined region around the top site than for the vdW-DF2-PES, for which this region extends towards the bridge sites. This behavior is related to the long range attractive vdW interaction accounted by the vdW-DF2-PES. As can be inferred from the figure, the repulsive area at distances around $3 \AA<Z<4 \AA$ covers the hollow and bridge sites for both the PW91-PES and the vdW-DF2-PES with a reduction of surface area in the latter case. The analysis of the dynamics at different energies has shown that it is, in fact, this long distance repulsive area the responsible for the non-monotonic behavior at low values of $E_{i}$ in the case of the PW91-PES. At collision energies around and below $10 \mathrm{meV}$, forces in $X$ and $Y$ contribute to drive efficiently molecules towards the top site, where the potential barrier is not present. When increasing the collision energy the available time for this lateral movement is reduced. In this way, as shown in the figure, for an incident energy of $40 \mathrm{meV}$ a significant amount of molecules are not able to move towards the top site and, as a consequence, they are reflected far from the surface resulting in a relatively low total sticking probability. Finally, for higher energies the molecules can overcome all the 


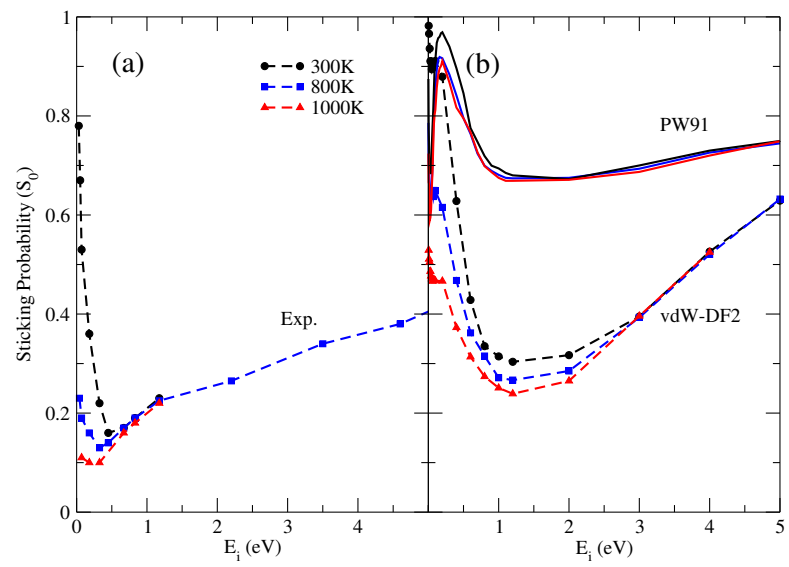

Fig. 3 (a) Experimental sticking probability at 300,800 , and $1000 \mathrm{~K}$ under normal incidence as function of the impact energy $E_{i}$. Data are extracted from 35 . (b) Probabilities obtained with the vdW-DF2 (dashed lines) and PW91 (solid lines) functionals within the GLO model for the same temperatures.

long distance barriers, and the reactivity, ruled by short distance region, is high. On the contrary, in the case of the vdW-DF2-PES, due to the long range attractive vdW forces, most of the molecules can get close to the surface irrespectively of the incident energy and, therefore, dynamics at large distances become unimportant. Due to this, reactivity decreases monotonically with energy and its low values are related to the more repulsive character of this PES at short distances.

As stated before, we have employed a GLO model that has showed to be a quite reasonable approximation in a variety of situations ${ }^{25 \mid 30[46] 60}$ in order to reach a better comparison of sticking probabilities between theoretical simulations and experimental measurements. In Fig. 3 we show the experimental results for $S_{0}$ as a function of energy for normal incidence conditions 35 together with the results of our GLO simulations using both the PW91-PES and the vdW-DF2-PES. Results are shown for three different temperatures, namely 300 , 800 , and $1000 \mathrm{~K}$. The total adsorption probability includes both dissociative adsorption and nondissociative adsorption. However, as the surface temperature increases and for both functionals, nondissociative adsorption becomes negligible for all energies. Therefore, the most interesting case is when $\mathrm{T}=300 \mathrm{~K}$, where, in the case of PW91, nondissociative adsorption amounts up to one half of the total adsorption at the lowest energies and it decreases monotonously reaching values below $2 \%$ at $200 \mathrm{meV}$. For the vdW-DF2 at the same temperature, all the adsorption is nondissociative at low energies, and it decreases as the energy and the dissociative adsorption increase, reaching a point where adsorption is equally shared by both contributions at around $800 \mathrm{meV}$.

Clearly, Fig. 3 shows that the results obtained with the vdWDF2-PES are in much better agreement with experiments, both qualitatively and quantitatively, than the ones obtained with the PW91-PES. Apart from the already extensively discussed behavior of $\mathrm{S}_{0}$ at low collision energies obtained with the PW91-PES and not observed in the experiments, the adsorption probabilities are greatly overestimated by this functional. Moreover, the measured reduction of the adsorption probability with temperature at the lowest energies is well captured by the vdW-DF2-PES, whereas for the PW91-PES the adsorption probability hardly depends on the surface temperature. In spite of the unquestionable improvement achieved by the vdW-DF2-PES, the obtained results still overestimate the experimental adsorption probabilities. In this respect, a fact that should be considered in future works is that although the GLO has showed to be a quite reasonable approximation, in some cases, by performing ab initio Molecular Dynamics simulations it has been demonstrated that to account for independent atom movement can have an impact in the adsorption dynamics 60.62 .

In summary, we have constructed a new PES using the vdWDF2 EXC functional for the $\mathrm{N}_{2}+\mathrm{W}(100)$ system and we have tested it by performing quasiclassical molecular dynamics simulations. We have shown that the main shortcomings of the previously used PW91-PES are solved by this new PES. In particular, we have demonstrated that the non-monotonic dependency of the sticking probablity as a function of the incident energy when $\mathrm{E}_{i}<500 \mathrm{meV}$, not observed in experiments, was due to the lack of long range vdW forces in the old PES. In particular, we found that this behavior was due to the presence of energy barriers at around $3 \AA<\mathrm{Z}<4 \AA$ in the PW91-PES that prevent a significant number of molecules from approaching the surface at energies of around $40 \mathrm{meV}$. Since these barriers disappear when vdW forces are considered, the vdW-DF2-PES does not generate the non-monotonic behavior. Additionally, we have shown that being the vdW-DF2PES more repulsive at short distances than the PW91-PES, the large overestimation of the sticking probability by the previous PES is much reduced. In this respect, it is worth to mention that from the two nonactivated paths to dissociation identified when using the PW91-PES, only one remains nonactivated when using the vdW-DF2-PES. We want to emphasize that these differences between the two PESs at short distances from the surface, that are important to achieve a better quantitative agreement with the experiments, are due to the fact that the elementary components in these two functionals are different, and are not related to the presence of vdW forces in the vdW-DF2 functional. All in all, we have shown that the vdW-DF2-PES provides a significant improvement in the description of the $\mathrm{N}_{2}$ adsorption dynamics on W(100), and that the inclusion of vdW forces solves the long standing problem that constituted the large qualitative and quantitative disagreement that existed between previous theoretical calculations and molecular beam experiments.

\section{Acknowlegdments}

A.P.T acknowledges financial support by the University of Bordeaux. This work was conducted in the scope of the Transnational Common Laboratory "QuantumChemPhys: Theoretical Chemistry and Physics at the Quantum Scale" (ANR-10-IDEX03-02). J.I.J acknowledges financial support by the Gobierno Vasco-UPV/EHU project IT756-13, and the Spanish Ministerio de Economía y Competitividad (Grant No. FIS2016-76471-P).

\section{Conflicts of interest}

There are no conflicts to declare. 


\section{Notes and references}

1 A. Nilsson, L. G. M. Petersson and J. K. Nørskov, Chemical Bonding at Surface and Interfaces, Elsevier, Amsterdam, 2008.

2 G. A. Somorjai, Introduction to Surface Chemistry and Catalysis, Wiley, New York, 1994.

3 I. Chorkendorff and J. W. Niemantsverdriet, Concepts of Modern Catalysis and Kinetics, Wiley-VCH, Weinheim, 2003.

4 G. Ertl, J. Vac. Sci. Technol. A., 1983, 1, 1247-1253.

5 K. Honkala, A. Hellman, I. N. Remediakis, A. Logadottir, A. Carlsson, S. Dahl, C. H. Christensen and J. K. Nørskov, Science, 2005, 307, 555-558.

6 C. T. Rettner, D. J. Auerbach, J. C. Tully and A. W. Kleyn, J. Chem. Phys., 1996, 100, 13021-13033.

7 A. M. Wodtke, D. Matsiev and D. J. Auerbach, Prog. Surf. Sci., 2008, 83, 167214.

8 G. O. Sitz, Rep. Prog. Phys., 2002, 65, 1165-1193.

9 A. W. Kleyn, Chem. Soc. Rev., 2003, 32, 87-95.

10 K. Golibrzuch, N. Bartels, D. J. Auerbach and A. M. Wodtke, Annu. Rev. Phys. Chem., 2015, 66, 399-425.

11 L. B. F. Juurlink, D. R. Killelea and A. L. Utz, Prog. Surf. Sci., 2009, 84, 69-134.

12 G. R. Darling and S. Holloway, Rep. Prog. Phys., 1995, 58, 1595-1672.

13 A. Gross, Surf. Sci. Rep., 1998, 32, 291-340.

14 G. J. Kroes, Prog. Surf. Sci., 1999, 60, 1-85.

15 G. J. Kroes, A. Gross, E. J. Baerends, M. Scheffler and D. A. McCormack, Acc. Chem. Res., 2002, 35, 193-200.

16 Dynamics of Gas-surface Interactions, ed. R. D. Muiño and H. F. Busnengo, Springer, Heidelberg, 2013.

17 M. Alducin, R. D. Muiño and J. Juaristi, Prog. Surf. Sci., 2017, 92, 317-340.

18 P. Nieto, E. Pijper, D. Barredo, G. Laurent, R. A. Olsen, E.-J. Baerends, G.-J. Kroes and D. Farías, Science, 2006, 312, 86-89.

19 C. Diaz, E. Pijper, R. A. Olsen, H. F. Busnengo, D. J. Auerbach and G. J. Kroes, Science, 2009, 326, 832-834.

20 C. Díaz, J. K. Vincent, G. P. Krishnamohan, R. A. Olsen, G. J. Kroes, K. Honkala and J. K. Norskov, Phys. Rev. Lett., 2006, 96, 096102.

21 C. Díaz, J. K. Vincent, G. P. Krishnamohan, R. A. Olsen, G. J. Kroes, K. Honkala and J. K. Nørskov, J. Chem. Phys., 2006, 125, 114706.

22 Q. Liu, X. Zhou, L. Zhou, Y. Zhang, X. Luo, H. Guo and B. Jiang, J. Phys. Chem. C, 2018, 122, 1761-1769.

23 L. Martin-Gondre, C. Crespos, P. Larregaray, J. C. Rayez, B. van Ootegem and D. Conte, J. Chem. Phys., 2010, 132, 204501.

24 D. Farías, C. Díaz, P. Rivière, H. F. Busnengo, P. Nieto, M. F. Somers, G. J. Kroes, A. Salin and F. Martín, Phys. Rev. Lett., 2004, 93, 246104.

25 I. Goikoetxea, J. Meyer, J. I. Juaristi, M. Alducin and K. Reuter, Phys. Rev. Lett., 2014, 112, 156101.

26 K. R. Geethalakshmi, J. I. Juaristi, R. Díez Muiño and M. Alducin, Phys. Chem. Chem. Phys., 2011, 13, 4357-4364.

27 R. Petuya, P.-A. Plotz, C. Crespos and P. Larregaray, J. Phys. Chem. C, 2014, 118, 21904-21910.

28 G.-J. Kroes, C. Díaz, E. Pijper, R. A. Olsen and D. J. Auerbach, Proc. Nat. Acad. Sci., 2010, 107, 20881-20886.

29 G.-J. Kroes, J. I. Juaristi and M. Alducin, J. Phys. Chem. C, 2017, 121, 1361713633.

30 L. Martin-Gondre, M. Alducin, G. A. Bocan, R. Díez Muiño and J. I. Juaristi,
Phys. Rev. Lett., 2012, 108, 096101.

31 M. Alducin, R. Díez-Muiño, H. F. Busnengo and A. Salin, Phys. Rev. Lett., 2006, 97, 056102 .

32 G. Volpilhac and A. Salin, Surf. Sci., 2004, 556, 129-144.

33 J. P. Perdew, J. A. Chevary, S. H. Vosko, K. A. Jackson, M. R. Pederson, D. J. Singh and C. Fiolhais, Phys. Rev. B, 1992, 46, 6671-6687.

34 C. T. Rettner, E. K. Schweizer, H. Stein and D. J. Auerbach, Phys. Rev. Lett., 1988, 61, 986-989.

35 C. T. Rettner, H. Stein and E. K. Schweizer, J. Chem. Phys, 1988, 89, 3337-3341.

36 C. T. Rettner, E. K. Schweizer and H. Stein, J. Chem. Phys., 1990, 93, 1442-1454.

37 M. Beutl, K. Rendulic and G. Castro, Surf. Sci., 1997, 385, 97-106.

38 J. I. Juaristi, M. Alducin, R. D. Muiño, H. F. Busnengo and A. Salin, Phys. Rev Lett., 2008, 100, 116102

39 I. Goikoetxea, J. I. Juaristi, M. Alducin and R. Díez-Muiño, J. Phys. Condens. Matter, 2009, 21, 264007.

40 M. Alducin, R. Díez-Muiño, H. F. Busnengo and A. Salin, J. Chem. Phys., 2006, 125, 144705

41 M. Alducin, R. Díez, H. F. Busnengo and A. Salin, Surf. Sci., 2007, 601, 3726 3730 .

42 G. Bocan, R. Díez, M. Alducin and H. F. Busnengo, J. Chem. Phys., 2008, 128, 154704.

43 B. Hammer, L. B. Hansen and J. K. Nørskov, Phys. Rev. B, 1999, 59, 7413-7421.

44 L. Martin-Gondre, J. I. Juaristi, M. Blanco-Rey, R. D. Muiño and M. Alducin, J. Chem. Phys., 2015, 142, 074704.

45 D. Migliorini, F. Nattino and G.-J. Kroes, J. Chem. Phys., 2016, 144, 084702.

46 I. Lončarić, G. Füchsel, J. I. Juaristi and P. Saalfrank, Phys. Rev. Lett., 2017, 119 146101.

47 S. Kneitz, J. Gemeinhardt, H. Koschel, G. Held and H.-P. Steinrück, Surf. Sci., 1999, 433-435, 27-31.

48 S. Kneitz, J. Gemeinhardt and H.-P. Steinrück, Surf. Sci., 1999, 440, 307-320.

49 B. Riedmüller, I. Ciobîcā, D. Papageorgopoulos, B. Berenbak, R. van Santen and A. Kleyn, Surf. Sci., 2000, 465, 347-360.

50 K. Lee, E. D. Murray, L. Kong, B. I. Lundqvist and D. C. Langreth, Phys. Rev. B, 2010, 82, 081101.

51 S. A. Adelman, J. Chem. Phys., 1979, 71, 4471-4486.

52 J. C. Tully, J. Chem. Phys., 1980, 73, 1975-1985.

53 H. F. Busnengo, W. Dong and A. Salin, Phys. Rev. Lett., 2004, 93, 236103.

54 J. Hafner and G. Kresse, in The Vienna AB-Initio Simulation Program VASP: An Efficient and Versatile Tool for Studying the Structural, Dynamic, and Electronic Properties of Materials, ed. A. Gonis, A. Meike and P. E. A. Turchi, Springer US, Boston, MA, 1997, pp. 69-82.

55 H.-J. Ernst, E. Hulpke and J. P. Toennies, Phys. Rev. B, 1992, 46, 16081-16105

56 S. Titmuss, A. Wander and D. A. King, Chem. Rev., 1996, 96, 1291-1306.

57 H. F. Busnengo, A. Salin and W. Dong, J. Chem. Phys., 2000, 112, 7641-7651.

58 R. A. Olsen, H. F. Busnengo, A. Salin, M. F. Somers, G. J. Kroes and E. J. Baerends, The Journal of Chemical Physics, 2002, 116, 3841-3855.

59 G. Kresse, Phys. Rev. B, 2000, 62, 8295-8305.

60 F. Nattino, O. Galparsoro, F. Costanzo, R. D. Muiño, M. Alducin and G.-J. Kroes, The Journal of Chemical Physics, 2016, 144, 244708.

61 D. Novko, M. Blanco-Rey, J. I. Juaristi and M. Alducin, Phys. Rev. B, 2015, 92 201411.

62 D. Novko, M. Blanco-Rey, M. Alducin and J. I. Juaristi, Phys. Rev. B, 2016, 93 , 245435. 